\title{
An UHPLC-TOF-MS method for quantifying novel brominated anticancer compound bromophenol-thiosemicarbazone hybrid and its application to in vivo pharmacokinetic study
}

Xiuxue Li, $i^{1,2,3,4}$, Lijun Wang ${ }^{1,2,3,4}$, Xiaoling Jia ${ }^{1,2,3,4}$, Chuanlong Guo 1,2,3,4,5, Chao Li1 ${ }^{1,2,3,4}$, Jiajia Zhang ${ }^{1,2,3,4}$ and Dayong Shi ${ }^{1,2,3,4,6^{*}}$

\begin{abstract}
Background: Bromophenol-thiosemicarbazone hybrid was a novel synthetic brominated anticancer compound with two bromine atoms. Bromophenol-thiosemicarbazone hybrid showed considerable selective inhibitory activity against PARP1 $\left(\right.$ IC $\left._{50}=29.5 \mathrm{nmol} / \mathrm{L}\right)$. UHPLC-TOF-MS was used to establish a new method to quantify bromophenolthiosemicarbazone hybrid bio-samples for indispensable quantitation analysis in further extensive pre-clinical studies.

Methods: Chromatographic and mass spectrometry parameters were optimized for quantitative method establishment. Improved protein-precipitated method was applied to the extraction of bromophenolthiosemicarbazone hybrid in rat plasma samples. Furthermore, this proposed method was applied to an intravenous bolus dose to male rats.

Results: Mobile phase was consisted of water for $A$ and acetonitrile for $B$ with $25 \mathrm{mmol} / \mathrm{L}$ formic acid in both $\mathrm{A}$ and $\mathrm{B}$. The flow rate was $0.30 \mathrm{~mL} / \mathrm{min}$, and the run time of bromophenol-thiosemicarbazone hybrid was $4.0 \mathrm{~min}$. A Thermo Fisher Accucore 2.6 um C18 column ( $50 \times 2.1 \mathrm{~mm}$ i.d.; San Jose, USA) was used for chromatographic separation. High resolution mass spectrometry was used to quantify samples by exact mass number of compound which was operated on negative ionization mode. Linear dynamic range of the established method was widely with $13.7-10000 \mathrm{nmol} / \mathrm{L}$. Pharmacokinetics properties of bromophenol-thiosemicarbazone hybrid were shown in the results.
\end{abstract}

Conclusion: This method was reliable and reproducible from sample preparation to analysis and storage stability under the investigated conditions. It may be useful for analysis of halogenated compounds and brominated compounds in ultra-performance liquid chromatography-mass spectrometry.

Keywords: Novel compound, Quantitation, UHPLC-TOF-MS, Pharmacokinetics

\footnotetext{
* Correspondence: shidayong@sdu.edu.cn

'Key Laboratory of Experimental Marine Biology, Institute of Oceanology,

Chinese Academy of Sciences, Qingdao, People's Republic of China

${ }^{2}$ Laboratory for Marine Drugs and Bioproducts of Qingdao National

Laboratory for Marine Science and Technology, Qingdao, People's Republic

of China

Full list of author information is available at the end of the article
}

\section{Springer Open}

(c) The Author(s). 2020 Open Access This article is licensed under a Creative Commons Attribution 4.0 International License, which permits use, sharing, adaptation, distribution and reproduction in any medium or format, as long as you give appropriate credit to the original author(s) and the source, provide a link to the Creative Commons licence, and indicate if changes were made. The images or other third party material in this article are included in the article's Creative Commons licence, unless indicated otherwise in a credit line to the material. If material is not included in the article's Creative Commons licence and your intended use is not permitted by statutory regulation or exceeds the permitted use, you will need to obtain permission directly from the copyright holder. To view a copy of this licence, visit http://creativecommons.org/licenses/by/4.0/. 


\section{Introduction}

Malignancies were becoming an increasing threat to world health and presenting various diversifications. Halogenated compounds exerted kinds of potent pharmacological activities, like anti-inflammatory, anti-oxidative, anticancer, and antimicrobial (Wang, Gloer et al. 2013). Bromophenol-thiosemicarbazone hybrid was a kind of novel synthetic brominated anticancer compound, with two bromine atoms. Pharmacological results showed that bromophenol-thiosemicarbazone hybrid showed considerable better inhibitory activity of PARP1 $\left(\mathrm{IC}_{50}=29.5 \mathrm{nmol} /\right.$ L) (Guo, wang et al. 2019). Generally, most naturally occurring bromine consists of a mixture of two stable isotopes: ${ }^{79} \mathrm{Br}$ and ${ }^{81} \mathrm{Br}$ (Heumann, Smith et al. 1998). The mass spectrum of the compound with one or two bromine atoms would show more than one precursor ions. Because of containing two bromine atoms, the exact mass spectrometry of bromophenol-thiosemicarbazone hybrid showed three exact mass numbers with 2 molecular weight differences, 393.8635, 395.8612, and 397.8590.

Ultra-high-performance liquid chromatography coupled with time-of-flight mass spectrometry (UHPLC-TOF-MS) method has not been reported for estimation of bromophenolthiosemicarbazone hybrid in rat plasma. High resolution mass spectrum could provide exact mass number data of compounds. In addition with sensitivity and accuracy of advanced mass spectrum, rapid, high sensitivity quantitative analysis of UHPLC-TOF-MS methods would be attainable. This method proposed a new rapid, sensitive UHPLC-TOF-MS method for quantifying brominated compound bromophenolthiosemicarbazone hybrid in rat plasma. This method was proposed through a precipitated sample preparation with the lower limit of detection of $13.7 \mathrm{nmol} / \mathrm{L}$ and a total chromatographic run time of $4 \mathrm{~min}$ for one injection. Bromophenolthiosemicarbazone hybrid plasma samples had good stability and negligible carryover and matrix effects under investigated conditions. This method may be useful for bio-sample analysis of bromophenol-thiosemicarbazone hybrid and analysis method development for brominated compounds.

\section{Materials and methods Materials}

Bromophenol-thiosemicarbazone hybrid was synthesized and purified in our lab. The purity was over $98 \%$. Formic acid of LC-MS-grade was obtained from Merck (Darmstadt, Germany). Acetonitrile, methanol, and the other analytical agents in HPLC-grade were all obtained from Honeywell (NJ, USA). Deionized water was generated by Milli $Q$ water purification instrument with model number of DIRCCT8 (Bedford, MA, USA).

\section{Analysis instruments and their conditions}

Analyses of chromatographic were executed using an Ultimate 3000 UHPLC system of Dionex (Sunnyvale, USA) which was consisted of an Ultimate 3000 Degasser, Pump, RS Autosampler, and column compartment. An Impact $\mathrm{HD}^{\mathrm{TM}}$ benchtop UHR-Q-ToF (Bruker Daltonik, Bremen, Germany) was interfaced with the UHPLC system. Chromatographic separation of bromophenolthiosemicarbazone hybrid was achieved on a Thermo Fisher Accucore $2.6 \mu \mathrm{m} \mathrm{C18}$ column $(50 \times 2.1 \mathrm{~mm}$ i.d.; San Jose, USA). Mobile phase used in this study included water (phase A, $25 \mathrm{mM}$ formic acid) and acetonitrile (phase B, $25 \mathrm{mM}$ formic acid). The flow rate in this method was $0.30 \mathrm{~mL} / \mathrm{min}$. The gradient elution regimen was starting at $2 \% \mathrm{~B}$ after sample introduction, and changing to $98 \%$ B after 0.1 min. After keeping 98\% B for 1.9 min, phase B was decreased back to $2 \%$ B and balanced for another $1.9 \mathrm{~min}$. The temperature of column oven and auto-sampler oven were maintained at 40 and $6{ }^{\circ} \mathrm{C}$, respectively. Mass spectrum parameters of bromophenolthiosemicarbazone hybrid were optimized to achieve the highest intensities. Bromophenol-thiosemicarbazone hybrid calibration curve samples were conducted by peak area ratio $(\mathrm{Y})$ against the corresponding nominal plasma concentrations of the analytes $(\mathrm{X}, \mathrm{nmol} / \mathrm{L})$ using $1 / \mathrm{X}$ weighted linear regressions.

\section{Calibration curves and quality controls}

Stock solution of bromophenol-thiosemicarbazone hybrid ( $1 \mathrm{mM})$ was used to construct calibration curves of samples by spiking with rat blank plasma. Standard plasma samples were generated by continuously diluted mode; the spiked concentration of calibration curves were 10000 , 3333, 1111, 370, 123, 41.1, and $13.7 \mathrm{nmol} / \mathrm{L}$. The concentration of low, medium, and high quality control (QC) samples were set as $100 \mathrm{nmol} / \mathrm{L}, 1000 \mathrm{nmol} / \mathrm{L}$, and 10,000 $\mathrm{nmol} / \mathrm{L}$ based on the linear range of the calibration curves. Quality control (QC) samples were prepared in the same way of precipitation as calibration curves.

\section{Method validation}

Validation of this established method was carried out following the Chinese Pharmacopoeia guidance and the USFDA guidelines [https://www.fda.gov/media/70858/download]. The upper (ULOQ) and lower limits of quantification (LOQ) were defined and determined by the virtual prepared linear range of the corresponding concentrations for rat plasma quantification. Stability test was performed under conditions simulating the sample preparing process. Studies of bromophenol-thiosemicarbazone hybrid extraction efficiency and matrix effects in rat plasma were performed as the referenced method reported (Li, Liang et al. 2007). Samples of QC were prepared by stock solution of bromophenolthiosemicarbazone hybrid $(1 \mathrm{mM})$ spiked with blank rat plasma in sextuplicate on three different batches for the accuracy and precision study including the intra-batch and inter-batch. 


\section{Sample preparation}

Rat plasma $(20 \mu \mathrm{L})$ was extracted with acetonitrile/ methanol $(50: 50, \mathrm{v} / \mathrm{v})$ in volume proportion of $1: 2$ for 5 min to remove plasma proteins. Five microliters supernatant was delivered to the UHPLC-TOF-MS system for bromophenol-thiosemicarbazone hybrid quantitative analysis after centrifugation $(13000 \mathrm{~g})$ for $10 \mathrm{~min}$.

\section{Rat pharmacokinetic study}

The ARRIVE guidelines (Kilkenny, Browne et al. 2010, McGrath, Lilley et al. 2015) were applied to this rat pharmacokinetic study. In addition, rat studies were carried out according to the care and use of laboratory animal guidelines established by Institute of Oceanology committees, Chinese Academy of Sciences. Animal sufferings were minimized by efforts. Three male Sprague-Dawley rats (250-290 g) were purchased from Jinan Pengyue Experimental Animal Breeding co., LTD (Jinan, China). Rats were allowed to accommodate in the breeding environment for 1 week before use, and the circumstance conditions were as follows: 12-h light/dark cycle, controlled temperature, and humidity in the range of $20-24^{\circ} \mathrm{C}$ and $30-70 \%$. Rats were fed filtered tap water and commercial rat chow in a freely intake way.

Rat blood sampling method was executed as reported (Hem, Smith et al. 1998). Serial blood samples $(\sim 100 \mu \mathrm{L})$ were collected through saphenous vein puncture into heparinized tubes before bromophenol-thiosemicarbazone hybrid dosage regimen and at 5, 15, $30 \mathrm{~min}$ and 1, 2, 4, 6, 8, 10 , and $24 \mathrm{~h}$ after an intravenous dose $(2 \mathrm{mg} / \mathrm{kg})$. Collected blood samples were immediately centrifuged to obtain the plasma fractions. Plasma samples were stored at $-70{ }^{\circ} \mathrm{C}$ until analysis. After use, experimental animals were put into euthanasia by a caudal venous air embolism. Noncompartmental model was selected in the calculation of pharmacokinetic parameters by Kinetica 2000 (version 3.0; Thermo Scientific, Philadelphia, PA, USA). The final conclusion data were showed as mean \pm standard deviation.

\section{Results and discussion Method development}

As bromophenol-thiosemicarbazone hybrid was a novel synthetic compound, an analytical method can facilitate the following step work in new drug development. The aim of this study was to develop a method to quantify bromophenolthiosemicarbazone hybrid in rat plasma on UHPLC-TOFMS instruments. The chromatographic column type and mobile phase were selected for the halogenated compound bromophenol-thiosemicarbazone hybrid. Successful chromatographic separation of bromophenol-thiosemicarbazone hybrid from the endogenous matrix effects in rat plasma samples was obtained on a Thermo Fisher Accucore $2.6 \mu \mathrm{m}$ C18 column $(50 \times 2.1 \mathrm{~mm}$ i.d.; San Jose, USA). $\log \mathrm{P}$ is one of the important physicochemical properties which determined the chromatographic retention behavior of the analytes (Kubik, Struck-Lewicka et al. 2015). CLogP value of bromophenol-thiosemicarbazone hybrid was 3.20 (calculated by Molinspiration Cheminformatics 2018) [http://www. molinspiration.com/cgi-bin/properties]. High bonding phase coverage of column used in this study can provide optimal retention for a range of hydrophobic analytes and was suitable for a variety of applications. Furthermore, the eluting capability of acetonitrile was more effective than methanol, so the acetonitrile was chosen as solvent B with $25 \mathrm{mM}$ formic acid, and water was for solvent A with $25 \mathrm{mM}$ formic acid. The optimized chromatographic conditions were constructed in a short run time of $4.0 \mathrm{~min}$ to achieve faster elution, improved resolution and separation from endogenous matrix in rat plasma, sufficient peak response and sharp peak shape. Twenty-five millimolar formic acid was added as electrolyte in mobile phase for better ionization of bromophenol-thiosemicarbazone hybrid. The retention time of bromophenol-thiosemicarbazone hybrid was 1.8 min under these chromatographic conditions.

Compound showed higher signal intensities in negative ion mode than positive mode with $1 \mu \mathrm{mol} / \mathrm{L}$ standard solutions. Therefore, the $[\mathrm{M}-\mathrm{H}]^{-}$ions were selected as parent ion for quantitation. As most naturally occurring bromine consists of a mixture of two stable isotopes: ${ }^{79} \mathrm{Br}$ and ${ }^{81} \mathrm{Br}$, the exact mass spectrometry of bromophenol-thiosemicarbazone hybrid, showed three exact mass numbers with 2 molecular weight differences, 393.8635, 395.8612, and 397.8590 in full scan mode for mass spectrum parameter optimization. The exact mass 395.8612 displayed higher intensity than 393.8635 and 397.8590; therefore, 395.8612 was used to quantify samples in our study. The structure and exact masses of bromophenolthiosemicarbazone hybrid are shown in Fig. 1. The mass spectrum source-dependent parameters were optimized to obtain the reproducible and adequate response of the compound. The optimized mass spectrum parameters of bromophenol-thiosemicarbazone hybrid in negative ion mode used for the measurement of the compound are showed in Table 1.

Negligible matrix effects and high recovery were achieved under organic reagents-based protein precipitation method for the test compound. Methanol/acetonitrile $(1: 1, \mathrm{v} / \mathrm{v})$ was chosen for bromophenol-thiosemicarbazone hybrid extraction in rat plasma samples. Mixed methanol and acetonitrile may get more efficient protein precipitation results and, therefore, bromophenol-thiosemicarbazone hybrid could acquire good adequate selectivity, peak shape, and sufficient response on mass spectrums analysis.

\section{Selectivity and chromatography}

The validated method showed good selectivity and suitable chromatography. Six individual lots of rat blank plasma were used in the method selectivity study. As shown in Fig. 2a, no endogenous compound was co- 


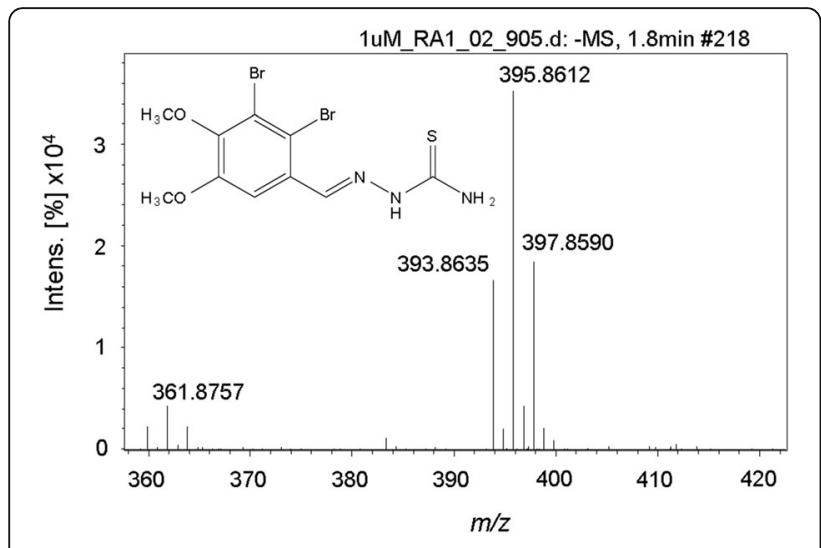

Fig. 1 Structure and exact mass of BTH in negative mode

eluted with peaks corresponding to bromophenolthiosemicarbazone hybrid. Peak response of limit of detection (LOD) was 3-fold higher than the rat blank plasma at the same retention time of compound bromophenolthiosemicarbazone hybrid in Fig. 2b. Therefore, the endogenous interference of rat plasma to bromophenolthiosemicarbazone hybrid was negligible. Figure $2 \mathrm{c}$ showed the chromatogram peak intensity for bromophenolthiosemicarbazone hybrid in plasma sample at $0.083 \mathrm{~h}$ after intravenous bolus dose regimen of $2 \mathrm{mg} / \mathrm{kg}$.

\section{Calibration curves, matrix effects, and extraction efficiency}

Correlation coefficients $(r)$ of rat plasma calibration curves was greater than 0.9999. The UHPLC-TOF-MS quantification linear dynamic range was $13.7-10000 \mathrm{nmol} / \mathrm{L}$. Matrix effects and extraction efficiency methods were performed as reported (Matuszewski, Constanzer et al. 2003). Briefly, set 1 , set 2 , and set 3 samples were prepared by spiking by stock solutions in organic reagent, extracted plasma endogenous matrix, and rat blank plasma. Organic reagent in set 1 was composed of methanol:acetonitrile $=$ $1: 1(\mathrm{v}: \mathrm{v})$, and extracted plasma endogenous matrix in set 2 was produced through removing plasma protein from rat blank plasma by organic reagent (same as in set 1 ) in the ratio of 1:2 (v:v). Samples of set 1 and set 2 were introduced to UHPLC-TOF-MS for analysis after preparation, while samples of set 3 were then precipitated by organic

Table 1 Optimize mass spectrum parameters for bromophenolthiosemicarbazone hybrid

\begin{tabular}{llll}
\hline Mass Parameters & Value & Mass Parameters & Value \\
\hline Capillary & $4000 \mathrm{~V}$ & Dry heater & $200{ }^{\circ} \mathrm{C}$ \\
End plate offset & $-500 \mathrm{~V}$ & Dry gas & $5.0 \mathrm{~L} / \mathrm{min}$ \\
Charging voltage & $2000 \mathrm{~V}$ & Scan range & $50-700 \mathrm{~m} / \mathrm{Z}$ \\
Nebulizer & $0.8 \mathrm{bar}$ & & \\
\hline
\end{tabular}

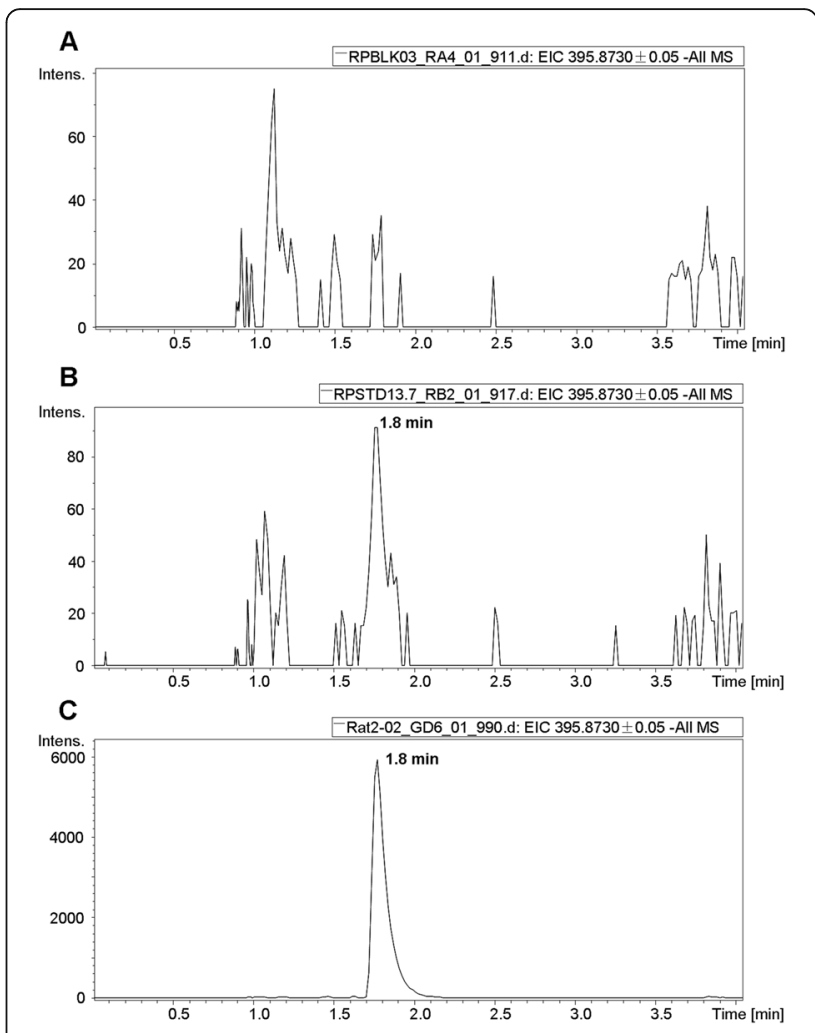

Fig. 2 Typical MS1 chromatograms of BTH in a blank plasma, b LOD sample, and $\mathbf{c}$ real subject sample of $0.083 \mathrm{~h}$ after intravenous injection of $2 \mathrm{mg} / \mathrm{kg}$ dose of

bromophenol-thiosemicarbazone hybrid

reagent the same as in set 1 in the ratio of $1: 2$ (v:v) after preparation. Extraction efficiency (\%) was determined by the formula of $3 \times($ set $3 /$ set 2$) \times 100$, and matrix effect (\%) was calculated by (set $2 /$ set 1$) \times 100$. Matrix effects and extraction efficiency studies were all presented through low, medium, and high-concentration levels $(n=$ 3 ). As shown in Table 2, the mean value of extraction efficiency of bromophenol-thiosemicarbazone hybrid was found to be $108-119 \%$, with coefficient of variation less than $11.0 \%$. The plasma matrix constitutions in organic reagent extraction had negligible ionization changes of bromophenolthiosemicarbazone hybrid retention time. The mean matrix effects of bromophenol-thiosemicarbazone hybrid were included in the range of 73.5-115\%, and the coefficients of variations were ranging below $13.0 \%$.

\section{Accuracy and precision of intra-batch and inter-batch}

Low-, medium-, and high-concentration quality controls were evaluated in the inter-batch and intra-batch rat plasma samples. All the precision results included in this study were evaluated through the calculation of \% RSD. In the nominal quality control concentration levels, as shown in Table 3, the mean accuracy of intra-batch and inter-batch samples were in the range of $108-115 \%$ and 
Table 2 Extraction efficiency $(n=3)$ and matrix effects $(n=3)$ for the analysis of bromophenol-thiosemicarbazone hybrid in rat plasma

\begin{tabular}{lll}
\hline $\begin{array}{l}\text { Nominal } \\
\text { concentration } \\
\text { (nmol/L) }\end{array}$ & Extraction efficacy (\%) & Matrix effects (\%) \\
\hline 100 & Mean \pm SD (RSD) \pm SD (RSD) \\
1000 & $108 \pm 12(11)$ & $73.5 \pm 9.6(13)$ \\
10000 & $114 \pm 2(2)$ & $110 \pm 3(2)$ \\
\hline
\end{tabular}

96.8-112\%, respectively. The corresponding precision values were all below $15 \%$, and this result showed acceptable accuracy and precision value of intra-batch and inter-batch.

\section{Stability study}

Rat plasma stability study was evaluated by comparing the detected nominal concentration of samples and samples suffer various given conditions. The investigated conditions included bench top storing stability under room temperature, autosampler maintaining at $6{ }^{\circ} \mathrm{C}$, stability of short- and long-term storage, and freeze/thaw cycles stability. As shown in Table 4, the concentration detected was 93.6-114\% of corresponding spiked concentrations after $3 \mathrm{~h}$ bench top storage period at room temperature. After $24 \mathrm{~h}$ storage under $6{ }^{\circ} \mathrm{C}$ in the autosampler, rat plasma samples were almost stable after plasma protein-removing with accuracy of 97.8-120\%. Although rat plasma samples were processed immediately in this study, freeze-thaw cycles stability of bromophenol-thiosemicarbazone hybrid was still investigated for three times for the application of our method to other studies with accuracy of $98.8-110 \%$. Short-term stability accuracy of bromophenol-thiosemicarbazone hybrid storing under $-70{ }^{\circ} \mathrm{C}$ for 3 days was in the range of $84.1-112 \%$ in the current study, and the long-term stability accuracy was $90.3-113 \%$ at $-70{ }^{\circ} \mathrm{C}$ for 40 days.

\section{Dilution, carryover, and system stability}

Based on the wide range of dynamic linear range of calibration curve of $13.7-10,000 \mathrm{nmol} / \mathrm{L}$, dilution of bromophenol-thiosemicarbazone hybrid may still be needed in other relative study although high plasma concentration was achieved in the intravenous dosage regimen. Dilution integrity test was carried out 2 times $(20000 \mathrm{nmol} / \mathrm{L})$ and $4(40000 \mathrm{nmol} / \mathrm{L})$ times, and the corresponding accuracies were $99.5 \%$ and $102 \%$ in this study with precision of $5.5 \%$ and $8.4 \%$, respectively. Future development and application to other studies of bromophenol-thiosemicarbazone hybrid-related sample diluting preparation may be indispensable.

Carryover study was following the standards at LOQ $(41.1 \mathrm{nmol} / \mathrm{L})$ and ULOQ $(10000 \mathrm{nmol} / \mathrm{L})$. Peak areas of blank plasma in the retention time of bromophenolthiosemicarbazone hybrid were $4.24 \%$ of their LOQs and $0.18 \%$ of their ULOQs, respectively. These results indicated that carryover between high and low concentration were negligible, and the elution of the compound from the column was facilitate. Even so, the sequences of sample analysis were still performed in a concentrationaugmenting mode in this study. The precision of system stability was $8.46 \%$, showing good mass spectrum signal uniformity in continuous sample reinjection $(n=6)$.

\section{Pharmacokinetic study}

This UHPLC-TOF-MS method which was established and validated was applied to a pharmacokinetic (PK) study intravenously administered into male rats $(n=3)$. As Fig. 3 displayed, the mean maximum plasma concentration $\left(C_{\max }\right)$ of bromophenol-thiosemicarbazone hybrid was $1300 \pm 10$ $\mathrm{nmol} / \mathrm{L}$ and attained at $0.08 \pm 0.00 \mathrm{~h}$ in rats. After dosing, bromophenol-thiosemicarbazone hybrid had lower systemic exposure level (indicated by $\mathrm{AUC}_{0-\infty}$ ), with value of $905 \pm$ $104 \mathrm{nmol} / \mathrm{L} \cdot \mathrm{h}$. Bromophenol-thiosemicarbazone hybrid eliminated rapidly after administration. The mean elimination half-life time $\left(t_{1 / 2}\right)$ was $0.75 \pm 0.04 \mathrm{~h}$. This short elimination half-life was attributed to the rapid elimination in vivo. In addition, this result was consistent with the high clearance of $5.32 \pm 0.51 \mathrm{~L} / \mathrm{h} / \mathrm{kg}$, which was larger than hepatic blood flow with value of $3.31 \mathrm{~L} / \mathrm{h} / \mathrm{kg}$ but smaller than cardiac output with value of $17.8 \mathrm{~L} / \mathrm{h} / \mathrm{kg}$. Thus, bromophenolthiosemicarbazone hybrid may have extra-hepatic clearance in rats. Volumes of distribution at steady-state $\left(V_{\mathrm{SS}}\right)$ was 5.77 $\pm 0.82 \mathrm{~L} / \mathrm{kg}$, and this result was far more larger than rat body fluid volume $(0.67 \mathrm{~L} / \mathrm{kg})$, which suggests that bromophenol-

Table 3 Intra-batch $(n=6)$ and inter-batch $(n=6)$ accuracy and precision for the analysis of bromophenol-thiosemicarbazone hybrid in rat plasma.

\begin{tabular}{|c|c|c|c|c|c|c|c|}
\hline \multirow{2}{*}{$\begin{array}{l}\text { BTH-2 } \\
\text { Analyte }\end{array}$} & \multirow{2}{*}{$\begin{array}{l}\text { Nominal } \\
\text { Conc. } \\
\text { (nmol/ } \\
\text { L) }\end{array}$} & \multirow[b]{2}{*}{ Conc. detected (nmol/L) } & \multirow{2}{*}{$\begin{array}{l}\text { Intra-batch data (\%) } \\
\text { Accuracy (mean } \pm \text { SD) }\end{array}$} & \multirow[b]{2}{*}{ Precision } & \multicolumn{3}{|l|}{ Inter-batch data (\%) } \\
\hline & & & & & Conc. detected (nmol/L) & Accuracy (mean \pm SD) & Precision \\
\hline LOQ & 41.1 & $44.4 \pm 2.3$ & $108 \pm 6$ & 5.3 & $44.9 \pm 5.0$ & $109 \pm 12$ & 11.2 \\
\hline LQC & 100 & $108 \pm 11$ & $109 \pm 11$ & 10 & $94.7 \pm 14.8$ & $96.8 \pm 14.8$ & 15.0 \\
\hline $\mathrm{MQC}$ & 1000 & $1125 \pm 53$ & $113 \pm 5$ & 5 & $1123 \pm 52$ & $112 \pm 5$ & 5 \\
\hline $\mathrm{HQC}$ & 10,000 & $11522 \pm 375$ & $115 \pm 4$ & 3 & $10789 \pm 765$ & $108 \pm 8$ & 7 \\
\hline
\end{tabular}


Table 4 Stability data for bromophenol-thiosemicarbazone hybrid in rat plasma $(n=3)$.

\begin{tabular}{|c|c|c|c|c|}
\hline Nominal conc. (nmol/L) & & 100 & 1000 & 10000 \\
\hline \multirow[t]{2}{*}{ On bench top at room temperature for $3 \mathrm{~h}$} & Conc. (nmol/L) & $93.6 \pm 14.2$ & $1139 \pm 36$ & $11196 \pm 622$ \\
\hline & Stability (\%) & 93.6 & 114 & 112 \\
\hline \multirow[t]{2}{*}{ In autosampler maintained at $6^{\circ} \mathrm{C}$ for $24 \mathrm{~h}$} & Conc. (nmol/L) & $97.8 \pm 15.6$ & $1128 \pm 63.6$ & $12023 \pm 1112$ \\
\hline & Stability (\%) & 97.8 & 113 & 120 \\
\hline \multirow[t]{2}{*}{ After 3 freeze/thaw cycles of $-70 \leftrightarrow 23^{\circ} \mathrm{C}$} & Conc. (nmol/L) & $98.8 \pm 3.1$ & $1095 \pm 32$ & $10965 \pm 683$ \\
\hline & Stability (\%) & 98.8 & 110 & 110 \\
\hline \multirow[t]{2}{*}{ Short term stability ( $-70^{\circ} \mathrm{C}$ for 3 days) } & Conc.(nmol/L) & $84.1 \pm 7.4$ & $1122 \pm 7$ & $11230 \pm 563$ \\
\hline & Stability (\%) & 84.1 & 112 & 112 \\
\hline \multirow[t]{2}{*}{ Long term stability ( $-70^{\circ} \mathrm{C}$ for 30 days) } & Conc.(nmol/L) & $92.0 \pm 4.6$ & $1095 \pm 78$ & $10626 \pm 768$ \\
\hline & Stability (\%) & 90.3 & 113 & 112 \\
\hline
\end{tabular}

thiosemicarbazone hybrid may have extensive distribution in rats.

The ClogP value of bromophenol-thiosemicarbazone hybrid was 3.2 calculated by molinspiration [http://www. molinspiration.com/cgi-bin/properties]. In light of, the LLOQ of this method was $13.7 \mathrm{nM}$, the free concentration in rat plasma expected may be very low based on the generally high plasma protein binding ratio of most of drugs which have high ClogP value. Compared to the agent's inhibitory concentration at $50 \%$ of efficacy (IC50) at 29.5 nM against PARP1, which was the free concentration of bromophenol-thiosemicarbazone hybrid, the LLOQ of $13.7 \mathrm{nM}$ may keep sensitive for EC50 studies in vivo (Ye, Nagar et al. 2016). In addition, bromophenol-thiosemicarbazone hybrid was expected to develop as an intravenous dosage form according to the results of IC50 in vitro in the future. Furthermore, if IC50 in vitro was $29.5 \mathrm{nM}$, the total plasma or tissue concentration was expected to be much higher in considering the possible high plasma/tissue protein binding ratio for bromophenol-thiosemicarbazone hybrid.

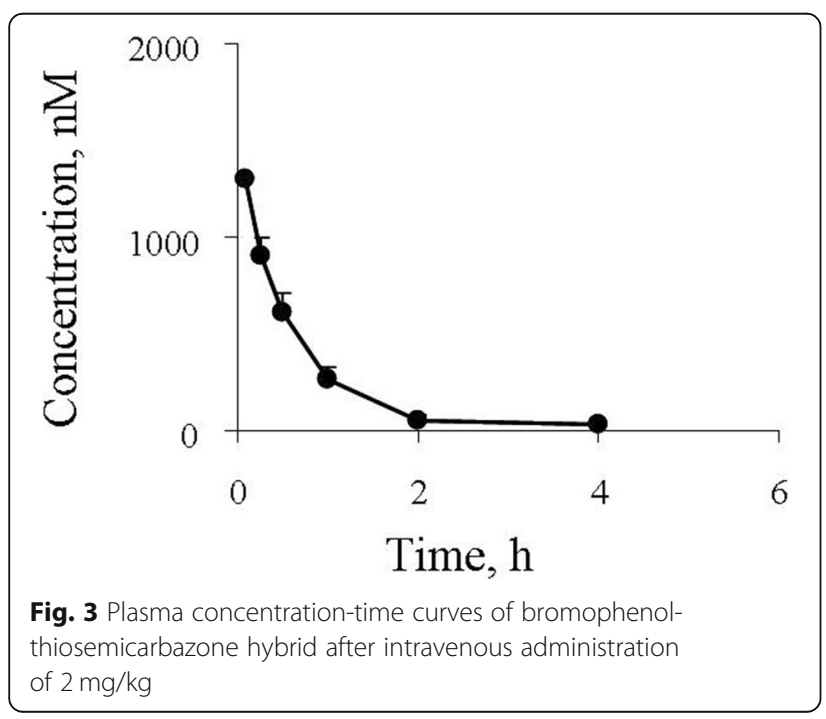

\section{Concluding remarks}

An UHPLC-TOF-MS method had been established and verified for rapid sensitive determination of new synthetic compound bromophenol-thiosemicarbazone hybrid in rat plasma. A wide linear dynamic range of 13.7-10,000 nmol/L was developed in this method. Despite of the fragment ion production, exact mass of precursor ion of bromophenol-thiosemicarbazone hybrid was used to quantify samples with the high throughput capabilities in analyzing bio-samples. Samples of stability and in vivo rat pharmacokinetic plasma samples were all analyzed based on this method. High throughput analysis of bromophenol-thiosemicarbazone hybrid in biosamples was feasible on account of the optimized short run time cycle of $4 \mathrm{~min}$. High sensitivity and wide linear dynamic range of this method will be very useful for applying in research and development of both oral and intravenous administration. As a kind of anticancer leading compound, bromophenol-thiosemicarbazone hybrid showed relatively moderate pharmacokinetic properties in vivo. This study also provided practical imitate to method development of halogenated compounds.

\section{Abbreviations \\ BTH: Bromophenol-thiosemicarbazone hybrid; AUC: Area under the concentration-time curve; $C_{\max }$ : Maximum plasma concentration; CLogP: Calculated octanol/water partition coefficient; Conc.: Concentration; HQC: High quality control; IC 50 : Half-inhibitory concentration; LQC: Low quality control; MQC: Medium quality control; PARP1: Poly(ADPribose) polymerase-1; PK: Pharmacokinetics; UHPLC: Ultra-high performance liquid chromatography; $t_{1 / 2}$ : Elimination half-life time; ULOQ: Upper limit of quantification; $V_{s s}$ : Distribution at stead state}

\section{Acknowledgements}

Not applicable in this section.

Authors' contributions

$\mathrm{XL}$ and DS designed the study. LW provided the compound of bromophenol-thiosemicarbazone hybrid. XL, LW, XJ, CG, CL, and JZ executed the animal studies. The UHPLC-TOF-MS analysis work was executed by XL and $\mathrm{XL}$, and DS supervised the experimental work and finished the draft of the manuscript. All authors read and approved the final manuscript. 


\section{Funding}

This work was supported by the National Natural Science Foundation of China (No. 81773586, 81872906, 81703354), the Key Research Program of Frontier Sciences, CAS, Grant NO.QYZDB-SSW-DQC014, Shandong Provincial Natural Science Foundation for Distinguished Young Scholars (JQ201722), and NSFC-Shandong Joint Fund Project (U1706213).

\section{Availability of data and materials}

The datasets used and/or analyzed during the current study are available from the corresponding author on reasonable request.

\section{Competing interests}

The authors declare that they have no competing interests.

\section{Author details}

'Key Laboratory of Experimental Marine Biology, Institute of Oceanology, Chinese Academy of Sciences, Qingdao, People's Republic of China. 2Laboratory for Marine Drugs and Bioproducts of Qingdao National Laboratory for Marine Science and Technology, Qingdao, People's Republic of China. ${ }^{3}$ Center for Ocean Mega-Science, Chinese Academy of Sciences, Qingdao, People's Republic of China. ${ }^{4}$ University of Chinese Academy of Sciences, Beijing, People's Republic of China. ${ }^{5}$ Department of Pharmacy, College of Chemical Engineering, Qingdao University of Science and Technology, Qingdao, People's Republic of China. 'State Key Laboratory of Microbial Technology, Shandong University, 72 Binhai Road, Qingdao 250100, Shandong, People's Republic of China.

Received: 28 September 2019 Accepted: 25 March 2020

Published online: 06 April 2020

\section{References}

Guo C, Wang L, Li X, Wang S, Yu X, Xu K, Zhao Y, Luo J, Li X, Jiang B, Shi D.

Discovery of novel bromophenol-thiosemicarbazone hybrid as potent selective inhibitors of poly(ADP-ribose) polymerase-1 (PARP-1) for use in cancer. J. Med. Chem. 2019;62:3051-67.

Hem A, Smith AJ, Solberg P. Saphenous vein puncture for blood sampling of the mouse, rat, hamster, gerbil, guinea pig, ferret and mink. Lab Anim. 1998;32: 364-8.

Heumann KG, Gallus SM, Rädlinger G, Vogl J. Precision and accuracy in isotope ratio measurements by plasma source mass spectrometry. J. Anal. At Spectrom. 1998:13:1001-8.

Kilkenny C, Browne W, Cuthill IC, Emerson M, Altman DG. National Centre for the Replacement, Refinement and Reduction of Amimals in Research, animal research: reporting in vivo experiments: the ARRIVE guidelines. $\mathrm{Br} J$ Pharmacol. 2010;160:1577-9.

Kubik $Ł$, Struck-Lewicka W, Kaliszan R, Wiczling P. Simultaneous determination of hydrophobicity and dissociation constant for a large set of compound by gradient reverse phase high performance liquid chromatography-mass spectrometry technique. J. Chromatogr A. 2015;1416:31-7.

Li L, Liang S, Du F, Li C. Simultaneous quantification of multiple licorice flavonoids in rat plasma. J. Am. Soc. Mass Spectrom. 2007;18:778-82.

Matuszewski BK, Constanzer ML, Chavez-Eng CM. Strategies for the assessment of matrix effect in quantitative bioanalytical methods based on HPLC-MS/MS. Anal Chem. 2003;75:3019-30.

McGrath JC, Lilley E. 2015. Implementing guidelines on reporting research using animals (ARRIVE etc.): new requirements for publication in BJP. Br J Pharmacol., 172: 3189-3193.

Wang BG, Gloer JB, Ji NY, Zhao JC. Halogenated organic molecules of Rhodomelaceae origin: chemistry and biology. Chem. Rev. 2013;113:3632-85.

Ye M, Nagar S, Korzekwa K. A physiologically based pharmacokinetic model to predict the pharmacokinetics of highly protein-bound drugs and the impact of errors in plasma protein binding. Biopharm Drug Dispos. 2016;37:123-41.

\section{Publisher's Note}

Springer Nature remains neutral with regard to jurisdictional claims in published maps and institutional affiliations.

\section{Submit your manuscript to a SpringerOpen ${ }^{\circ}$ journal and benefit from:}

- Convenient online submission

- Rigorous peer review

- Open access: articles freely available online

- High visibility within the field

- Retaining the copyright to your article

Submit your next manuscript at $\boldsymbol{\nabla}$ springeropen.com 